International Journal of

BioScience and Applications

\title{
Experience of local anesthetic systemic toxicity in a patient who underwent Cesarean delivery with spinal anesthesia after failed epidural painless delivery. -Case report-
}

\author{
First A. Hyun Joo Heo M.D. ${ }^{1}$, Second B. Ji Hye Lee M.D. ${ }^{*}$, Third Da Wa Jung
} M.D. ${ }^{1}$,Four C. Ji Hun Park M.D ${ }^{1}$, and Five Ayung Gu Cho M.D ${ }^{1}$

${ }^{1}$ Department of Anesthesiology and Pain Medicine, Presbyterian Medical Center, Jeonju, Korea

\begin{abstract}
Background/Objectives: Local anesthetic systemic toxicity (LAST) are life-threatening conditions in obstetric anesthesia. Methods/Statistical analysis: The case in which a patient who underwent Cesarean delivery had performed spinal anesthesia due to a change in the position of the epidural catheter. Findings: Patient had presented cardiovascular and neurologic symptoms relevant with LAST, and was treated promptly with intravenous lipid emulsion (ILE). Improvements/Applications: LAST can also occur in spinal anesthesia and can be effectively treated with ILE.
\end{abstract}

\section{Index Terms}

Intravenous lipid emulsion; Local anesthetics; Systemic; Toxicity

\footnotetext{
Corresponding author: Ji Hye Lee

o2ljh@hanmail.net

- Manuscript received January 15, 2020.

- Revised February 10, 2020 ; Accepted March 2, 2020.

- Date of publication March 30, 2020.

(C) The Academic Society of Convergence Science Inc.

2619-8363 @ 2020 IJBSA. Personal use is permitted, but republication/redistribution requires IJBSA permission.
} 


\section{INTRODUCTION}

This document is a Microsoft Word template for IJASC. Use of this document as a template is optional. If you are reading a paper version of this document, please download the electronic file from IJASC website so you can use it to prepare your manuscript. Authors may prepare their papers for review using any word processor, one or two columns, single or double spaced. Please follow the writing style specified in this document.

When you open the template, select "Print Layout" from the "View" menu in the menu bar (View > Print Layout). Epidural catheterization is one of the effective neuraxial block for labor pain [1]. Among patients who have undergone this epidural block, emergency Cesarean delivery may be performed for various reasons. However, general anesthesia or other neuraxial block must be performed for Cesarean delivery if epidural block is inadequate.

There are several complications represented by high neuraxial block, local anesthetic systemic toxicity (LAST), failed spinal anesthesia, and another when repeat regional block after inadequate epidural block. When high neuraxial block and LAST occur, it can show extraordinary cardiovascular and neurologic symptoms and can be life threatening [2].

We present a case of LAST which is difficult to distinguish with high neurxial block was developed during Cesarean delivery and effectively treated to intravenous lipid emulsion (ILE).

\section{CASE REPORT}

\section{A. Manuscripts submission}

The patient and her family gave written informed consent for the authors to announce this case. A 30-year-old woman, a primipara at 38 weeks 3 days gestation was admitted our obstetrics for induction of labor. height and weight of the patient were $158 \mathrm{~cm}, 57 \mathrm{~kg}$. Medical history, obstetric history and other allergy history were no remarkable. Furthermore, she had never been under anesthesia. Vital signs and laboratory values were within normal limits in delivery room.

After admission, the patient monitors were placed: electrocardiography (ECG), pulse oximetry, non-invasive blood pressure. Intravenous infusion started with crystalloid. In addition, tocodynamometer was applied for monitored fetal heart rate. A prostaglandin E2 vaginal suppository was used for induction of labor. Patient complained of labor pain and underwent epidural catheterization for painless delivery.

The patient's blood pressure was $100 / 60 \mathrm{mmHg}$, heart rate was 65 beats/min, respiratory rate was 20 times $/ \mathrm{min}$, and oxygen saturation was $98 \%$ before the procedure. Epidural catheterization was performed as follows; Patient had a position of left lateral. The back of patient was sterilized with $2 \%$ chlorhexidine and draped for aseptic technique. The L3-L4 intervertebral space was infiltrated with $2 \mathrm{~mL}$ of $2 \%$ lidocaine for skin and subcutaneous anesthesia. A 18-gauge Tuohy needle was inserted to find an epidural space with a loss of resistance technique. A loss of resistance was confirmed at $3 \mathrm{~cm}$ from the skin, and a 20-gauge catheter (Perifix ${ }^{\circledR}$ Epidural Anesthesia Catheter; B. Braun, Melsungen, Germany) was inserted to the epidural space at a depth of $7 \mathrm{~cm}$ from the skin. The epidural space was confirmed by negative pressure aspiration, there was no aspirates blood or cerebrospinal fluid (CSF). Thereafter, $3 \mathrm{~mL}$ of a mixed solution of $1.5 \%$ lidocaine and $1.5 \mu \mathrm{g}$ of epinephrine (1: 200,000) was administered for exclusion of accidental intravascular or subarachnoid space injection. During observation for five minutes, heart rate and blood pressure did not increase, the motor was not blocked, and the fetal heart rate was normal range. Then the catheter was fixed and the patient took a supine position. In order to reduce the pain, bolus of $6 \mathrm{~mL}$ (ropivacaine $0.15 \%$ and fentanyl $2 \mu \mathrm{g} / \mathrm{mL}$ ) was administered and patient-controlled epidural analgesia (PCEA) was connected. PCEA infusion (ropivacaine $0.15 \%$ and fentanyl $2 \mu \mathrm{g} / \mathrm{mL}$ ) was started with basal rate 6 $\mathrm{mL} / \mathrm{h}$, bolus $6 \mathrm{~mL}$, and lock out time $30 \mathrm{~min}$.

About 21 hours later an epidural catheterization, emergency Cesarean delivery was determined because of further labor did not progress due to cephalopelvic disproportion. Standard monitors were placed when the patient arrived in operation room. Vital signs were blood pressure $110 / 69 \mathrm{mmHg}$, heart rate 76 beats $/ \mathrm{min}$, respiratory rate 20 times $/ \mathrm{min}$, and oxygen saturation $98 \%$. Although total of $36.9 \mathrm{mg}$ of ropivacaine via PCEA was administered to epidural space of the patient for 21 hours, the patient complained of labor pain. When the position of the catheter was checked, the epidural catheter was protruding about $2 \mathrm{~cm}$ from the fixed location. The condition of the patient and fetus was stable, and there was no evidence of neuraxial block. Hence, we decided to perform spinal anesthesia.

Spinal anesthesia was performed as follows; The patient took left lateral position, and was sterilized with $2 \%$ chlorhexidine and draped. The L4-L5 intervertebral space was infiltrated with $2 \mathrm{~mL}$ of $2 \%$ lidocaine for skin and subcutaneous anesthesia because epidural catheter had inserted L3-L4. Using a 25-gauge pencil-point spinal needle (Pencan ${ }^{\circledR}$ pencil spinal needle, B. Braun, Melsungen, Germany), $0.5 \%$ bupivacaine $8 \mathrm{mg}$ with fentanyl $20 \mu \mathrm{g}$ was administered for spinal anesthesia. Then, an epidural catheter was removed. Patient was taken head up and left tilt. Oxygen was supplied via a simple facial mask $(5 \mathrm{~L} / \mathrm{min})$. Respiratory rate and end tidal $\mathrm{CO}_{2}$ was monitored. When the level of spinal anesthesia was up to T6 dermatome as measured by the pinprick test, operation was started. About 4 minutes later, she delivered a neonate with 1min and 5min Apgar scores of 9, and 10.

One minute later a delivery, the patient complained of headache, nausea, and vomiting. Vital signs were monitored; blood pressure was $90 / 50 \mathrm{mmHg}$, heart rate fluctuated between $80-95$ beats/min, respiratory rate was 15 times/min, end tidal $\mathrm{CO}_{2}$ was $27-30 \mathrm{mmHg}$, oxygen saturation was $100 \%$ and conduction delay was visible on ECG. Anesthesia level was immediately confirmed as T4 dermatome by pinprick test. Patient was kept monitoring 
circumspectly after head up, given $100 \%$ oxygen and administered phenylephrine $100 \mu \mathrm{g}$. About three minutes later, patient complained of feeling restless, weakness and dizziness, then patient was agitated and consciousness decreased (Ramsay scale was degraded from $1 / 6$ to $4 / 6$ ). Vital signs were unchanged compared to before this situation.

Treatment was forthwith commenced with ILE of 1.5 $\mathrm{mL} / \mathrm{kg}$ was administered within two minutes. Since then, it has been persistently settled at $0.25 \mathrm{~mL} / \mathrm{kg} / \mathrm{min}$. About five minutes after ILE treatment, the patient's mental was alerted (Ramsay scale was 1/6), other symptoms were improved, conduction abnormality on ECG disappeared. ILE was administered total $250 \mathrm{~mL}$. After Cesarean delivery, we circumspectly monitored about 2 hours in the post anesthetic care unit and no problems were found. Patient was handed over to ward and monitored. Patient reported no discomfort and was discharged 4 days later after operation.

\section{DISCUSSION}

Complications in the obstetric anesthesia such as sudden changes in consciousness, problems in the cardiovascular vascular system or respiratory system are associated with surgery, emotional stress, anaphylaxis or neuraxial anesthesia. For example, amniotic embolism, pulmonary embolism, cerebral infarction, and hemorrhage may also occur during Cesarean delivery. In addition, anxiety, hyperventilation syndrome, vasovagal response and anaphylaxis can also be occurred.

Before the occurrence of the problem condition, the patient did not find any abnormal findings in urine test, blood test, ECG and in physical examination. However, in the case of our patient, dyspnea, chest pain and severe hypotension do not appear and there is no significant change in end tidal $\mathrm{CO}_{2}$, respiratory rate during surgery. And there was a change in consciousness, but no other signs of brain infarction or hemmorrhage were suspected. The patient had no allergy in the past history and was able to eliminate the appearance of anaphylaxis symptoms such as urticaria, hypotension. Since symptoms were not displayed by the surgeon's manipulation, the case of vasovagal response could be excluded. For that reason, other differential diagnoses are less likely but possible to consider LAST or high neuraxial block.

Neuraxial block for obstetric anesthesia has several complications such as LAST, anaphylaxis, high neuraxial block, failed block, postdural puncture headaches, epidural abscess or meningitis, epidural hematoma, neurologic injury and another [2]. LASTs are rare, accounting for of $0.03 \%$. Pregnancy, on the other hand, increases the risk of LAST [3,4]. High neuraxial block incidence are unknown, but are occurred of 1:4,336 anesthesia at a retrospective study in the USA [2].

High neuraxial block is spread of local anesthetics affecting the spinal nerves above T4 or more intracranial that will occur in both spinal and epidural anesthesia. The clinical presenting features are depending on block level, such as severe hypotension, bradycardia, paresthesia in hands/arms, hypoventilation, respiratory arrest, slurred speech, or loss of consciousness [5]. Risk factors of high neuraxial block were obesity of patient, spinal procedure after failed epidural anesthesia, low height, epidural procedure after wet tap and spinal deformity [2]. Untreatment may ascend further leading to unfortunate consequences like a cardiac arrest [5].

LAST is commonly found in epidural anesthesia rather than spinal anesthesia. LAST are occurred in usually systemically absorbed or misinjected of local anesthetics, manifested features in central nervous system toxicity being displayed at lower concentrations than cardiovascular toxicity. Central nervous system toxicity signs are tongue numbness, tinnitus, and metallic taste, and when it gets worse, it can occur seizures or coma and respiratory arrest. The effects on cardiovascular system can come down to hypotension, bradycardia, as well as severe cardiac arrest by directly suppressing the cardiac contraction and even the nerve conduction of the heart. There are several risk factors that can generate LAST. Depending on the amount and potency of local anesthetics, the combination of vasoactive agent, the site of procedure, a patient's age, pregnancy, kidney, heart, and liver disease will be affected. The onset times at which LAST can occur are variable, but most often appear immediately to 60 minutes after local anesthetics administration. It may be delayed, especially if continuous infusion is performed [3$4,6]$. LAST and high neuraxial block are life threatening complications.

In the case of the patient, she was controlled labor pain with epidural block; moved to operation room for emergency Cesarean delivery. The epidural catheter position was changed during transfer from delivery room to operation room, and spinal anesthesia was performed and removed the catheter. She presented with cardiovascular and neurologic symptoms during surgery. Therefore, we first doubted high neuraxial block when the patient had a first symptoms as headache, nausea and conduction delay on ECG. However, she was invisible of symptoms that block spreading above $\mathrm{T} 4$ level or more such as paresthesia or numbness in hands/arms, respiratory difficulty, hypoventilation or desaturation, and was not seen more than T4 level checked with pinprick test [7-8].

The effect of ropivacaine on cardiovascular vascular collapse and central nervous system is lower than other local anesthetics. In addition, ropivacaine has vasoactive properties that has the effect of increase duration and delay systematic absorption. Tolerated dose of ropivacaine is $81.7 \pm 22.3 \mathrm{mg}$ in the volunteers [9-10]. The volume of ropivacaine used in the patient was within the safety range. However, the systemic toxicity of ropivacaine cannot be seen as much less than that of other local anesthetics, we cannot be convinced that it was not systematic absorption.

The epidural catheter tip was not seen blood when it was removed because of position changed. However, it is not possible to confirm that the epidural catheter has never been in position of intravascular when it changed. In addition, risk of local anesthetics systemic absorption increased in obstetric patient which blood vessel is engorged. It is also thought that local anesthetics are accidentally absorbed through PCEA.

The level of serum local anesthetics was not measured when symptoms appeared to the patient. Also, 12 lead ECG for accurate diagnosis was not acquired when is seen of conduction abnormality. The dermatome level was T4 when it was last checked with pinprick test before ILE 
administered, but it cannot be guaranteed that local anesthetics not spread above T4.

High nueraxial block and LAST requires prompt diagnosis and treatment. Management of high neuraxial block assesses the patient with airway, breathing, circulation and disability. In general, ILE is effectually for LAST management unlike the common cardiac arrest; furthermore, it requires assessment of airway, circulation and neurology [1-3]. ILE is used antidote for multiple drugs [11], and is used for local anesthesia reversal when high and prolonged neuraxial block in case reports [12-15]. In our patient, it is difficult to distinguish with LAST and high neuraxial block; nevertheless, ILE is considered to be an appropriate treatment.

In conclusion, LAST and high neuraxial block are conditions that can cause very serious complications such as cardiac arrest. LAST and high neuraxial block may appear simultaneously, and can be difficult to distinguish. When such a situation occurs, it is necessary to make a prompt decision and treat with ILE immediately.

\section{REFERENCES (APA STYLE)}

[1] R.E. Collis, D.W.L. Davies, W. Aveling. (1995) Randomised comparison of combined spinal-epidural and standard epidural analgesia in labour. The Lancet 345:1413-6.

[2] D’Angelo R. Smiley RM. Riley ET. Segal S. (2014). Serious complications related to obstetric anesthesia: the serious complication repository project of the Society for Obstetric Anesthesia and Perinatology. Anesthesiology 120(6):1505-12.

[3] Gitman M, Barrington MJ, MBBS, FANZCA. Local anesthetic systemic toxicity. (2018). A review of recent case reports and registries. Regional Anesthesia and Pain Medicine 2(43):124-30.

[4] B.D. Sites, et al (2012) Incidence of local anesthetic systemic toxicicty and postoperative neuraologic symptoms associated with 12,668 ultrasound-guided nerve blocks. An analysis from a prospective clinical registry. Regional Anesthesia and Pain Medicine 37(5):478-82.

[5] Einhorn LM, Habib AS. (2016). Evaluation of failed and high blocks associated with spinal anesthesia for Cesarean delivery following inadequate labour epidural: a retrospective cohort study. Can J Anaesth. 63(10):1170-8.

[6] Oh S, Chung J, Lee SM, Chung K, Kwon K. (2012). Late onset of systemic toxicity of local anesthetics in brachial plexus block - A case report-. Anesth Pain Med 7:372-4.

[7] S.Vaida, D. Cattano, D. Hurwitz, B. Mets. (2015) Algorithm for the anesthetic management of cesarean delivery in patients with unsatisfactory labor epidural analgesia. $\quad$ F1000research. 4:98 (doi:10.12688/f1000research.6381.1).

[8] KS Parikh, S Seetharamaiah. (2018) Approach to failed spinal anaesthesia for caesarean section. Indian J Anaesth 62:691-7.

[9] Dureau P, Charbit B, Nicolas N, Benhamou D, Mazoit JX. (2016). Effect of Intralipid ${ }^{\circledR}$ on the Dose of Ropivacaine or Levobupivacaine Tolerated by Volunteers: A Clinical and Pharmacokinetic Study. Anesthesiology. 125(3):474-83.
[10] M. Harvey, G. Cave. (2017) Lipid emulsion in local anesthetic toxicity. Curr Opin Anesthesiol 30;632-8.

[11] Rothschild L, Bern S, Oswald S, Weinberg G. (2010). Intravenous lipid emulsion in clinical toxicology. Scand J Trauma Resusc Emerg Med. 18:51.

[12] P.Toledo, H.C. Nixon, J.M. Mhyre, C.A. Wong, G. Weinberg. (2013) Availability of lipid emulsion in united states obstetric units. Anesthesia and analgesia. 166(2);406-8.

[13] Eldor J. (2018). Lipid emulsion of local anesthesia reversal (LAR) after prolonged spinal/epidural anesthesia. Jor Health Sci Development. 1(1):43-7.

[14] Eldor J. (2018). Local anesthesia reversal (LAR) of total spinal anesthesia (TSA) by lipofundin (Lipid Emulsion). 1(1);67-72.

[15] J.D.C. Lin, E. Sivanesan, T.T. Horlocker, A. Missair. (2017). Two for one: a case report of intravenous lipid emulsion to treat local anesthetic systemic toxicity in term pregnancy. A\&A case reports. 8:235-7. 